\title{
Determinación de los parámetros de crecimiento de la Tilapia Nilótica (Orechromis niloticus) en un estanque revestido con geomembrana y abastecido con agua de subsuelo.
}

\section{Determination of growth parameters of Nile tilapia (Orechromis niloticus) in a Magazine with geomembrane pond stocked with water and subsoil.}

\author{
Alex Garcia*, Juan Tume**, Víctor Juárez ${ }^{* * *}$
}

http://dx.doi.org/10.21503/CienciayDesarrollo.2012.v15i2.05

\section{RESUMEN}

En este trabajo se han evaluado los parámetros de crecimiento de la Tilapia Nilótica (Orechromis niloticus) en un estanque del instituto de investigación de la UAP-Piura, abastecida con agua del sub suelo, se hicieron los muestreos biométricos respectivos (Longitud - Peso), con un tamaño de muestra de 30 ejemplares por muestreo. Estos muestreos se realizaron por un tiempo de 3 meses, lo que permitió obtener como resultados los parámetros de crecimiento de la tilapia.

La velocidad de crecimiento semanal total durante la fase de cultivo de 22 semanas fue de $17.29 \%$ o de $7.66 \mathrm{gr}$ por semana llevados a crecimiento diario $1.09 \mathrm{gr}$. A una densidad de 5 peces $/ \mathrm{m}^{2}$.

Los parámetros de crecimiento fueron el L asintótico $29.93 \mathrm{~cm}$, el valor K 1.536/año, el $\mathrm{T}_{0}$ es -0.132 y el W asintótico 392.32 gr.

Palabras clave: Tilapia nilótica, agua de subsuelo.

\section{ABSTRACT}

In this work there have been evaluated the parameters of growth of the Tilapia Nilótica (Orechromis niloticus) in a reservoir of the institute of investigation of the UAP-Piura, supplied with water of the sub I occur, there were done the biometric respective samplings (Length - Weight), with a size of sample of 300 copies for sampling. These samplings realized in a time of 3 months, which allowed to obtain like proved the parameters of growth of the tilapia. The speed of weekly total growth during the phase of culture of 22 weeks was $17.29 \%$ or 7.66 gr per week taken to daily growth 1.09 gr. To a density of 5 fish $/ \mathrm{m}^{2}$. The parameters of growth were the L asintótico $29.93 \mathrm{~cm}$, the value $\mathrm{K} 1.536 /$ año, the T0 is- 0.132 and the W asintótico $392.32 \mathrm{gr}$.

Key words: Tilapia nilótica, agua de subsuelo.

\footnotetext{
* Ing. Magiter en Ingeniería Ambiental, Director de la Escuela de Ingeniería Ambiental de la UAP- Piura. E-mail: agarciacri@yahoo.com

** Ingeniero docente del área de extracción pesquera de la Facultad de Ingeniería Pesquera de la Universidad Nacional de Piura y de la Escuela de Ingeniería Ambiental de la UAP-Piura. E-mail: delfin_jtr@hotmail.com

${ }^{* * *}$ Ingeniero, Magister en Acuicultura, docente del área de acuicultura de la Facultad de Ingeniería Pesquera de la Universidad Nacional de Piura. E-mail: victorjuarezp@hotmail.com
} 


\section{INTRODUCCIÓN}

La Tilapia se encuentra catalogada dentro del grupo de peces con mayor futuro en cultivos comerciales ya que su periodo de crecimiento es relativamente más corto al de otras especies y presenta alta adaptabilidad a diferentes ambientes de producción (Arul, 2002; Granados y cols, 2002; Garduño, 1998; Green, 1995, Ward, 1995).

Meyer (1999) menciona que el crecimiento de los peces depende en gran parte de la calidad del agua; por lo que para lograr una buena producción, es necesario mantener las condiciones físico-químicas del agua dentro de los límites de tolerancia para la especie a cultivar. La Tilapia se considera una especie productiva promisoria por ser rústica (Castillo,2001; Wicki, 1998; Kitaev, 2002); y por esa característica se decidió trabajar con ella para realizar el presente estudio.

El estudio de los principales parámetros de crecimiento de la especie Tilapia del Nilo Oreochromis niloticus, aplicando la Estadística que evalúa los recursos pesqueros nos permitió analizar e interpretar el comportamiento de dicha especie en condiciones diferentes de sustrato

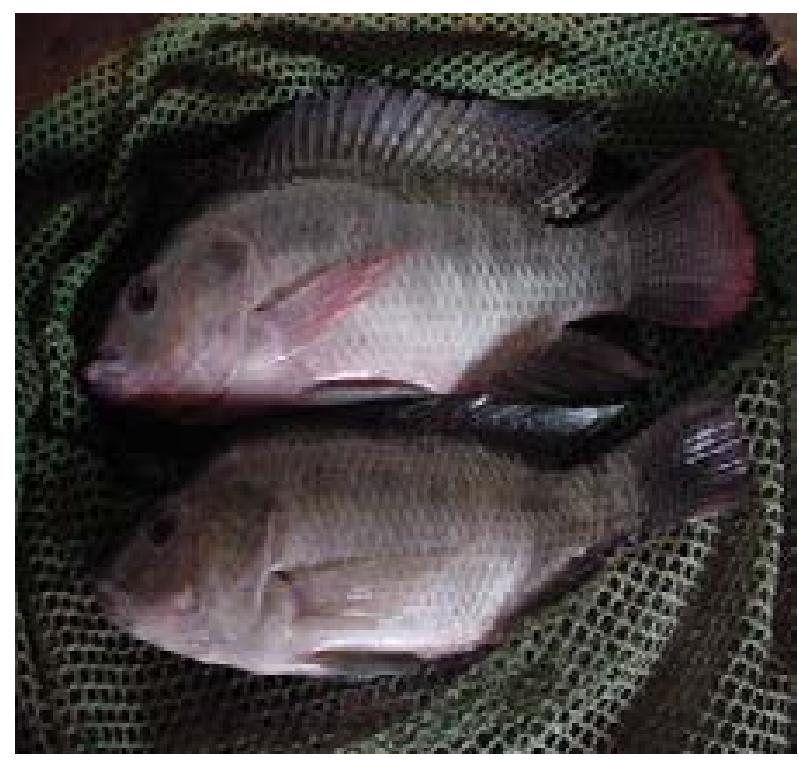

con estanque revestido con geomembrana y abastecido con agua del subsuelo en Piura

Por otro lado el estudio ayuda a Determinar la relación talla - peso para la especie Oreochromis niloticus Tilapia Gris o del Nilo en cultivo

\section{MATERIALES Y MÉTODOS}

El estudio se llevo a cabo en las instalaciones del centro de investigación de la Universidad Alas Peruanas. Filial Piura, en el km 3,5 carretera Piura-Chulucanas.

La toma de datos se realizó en un periodo de 148 días entre los meses de mayo y setiembre del 2011.

Se usó un diseño completamente al azar, en un estanque de tierra semiexcavado revestido con geomembrana, de forma rectangular con dimensiones de $50 \mathrm{~m} \times 20 \mathrm{~m}$ y $1.20 \mathrm{~m}$ de profundidad, con un sistema de suministro de agua del subsuelo por una electrobomba de 4 pulgadas. Apoyados con un aireador de paleta del tipo Taiwanés.

Se sembraron 5000 alevines de Oreochromis niloticus, los cuales fueron comprados ya revertidos sexualmente (masculinizados). La densidad de siembra fue de 5 alevines por $\mathrm{m} 3$. Los alevines tenían 30 días de nacidos y presentaban un peso promedio de $0,5 \mathrm{~g}$.

$\mathrm{Al}$ inicio del experimento los peces fueron alimentados dos veces al día a saciedad con alimento Puritilapia de la marca Purina, el análisis proximal marcado en la etiqueta fue de $50 \%$ de proteína, a los dos meses de edad se cambió la alimentación a base de un alimento comercial que contenía 30\% de proteína, con una alimentación racionada 3 veces al día en función a su crecimiento, con tasas de alimentación desde $25 \%$ hasta el $2 \%$ de la biomasa, al final de la cosecha.

Se suministro aireación por medio de un sistema 
artificial consistente en una aireador de 4 paletas del tipo Taiwanés con una potencia de $2 \mathrm{HP}$ ubicado en la parte media del estanque, en horas de la noche por lapso de 6 horas/día a partir del tercer mes de cultivo.

La toma de datos de peso y longitud, se realizó cada 7 días, para lo cual se utilizó una atarralla con malla de $18 \mathrm{~mm}$ de abertura. En cada toma de datos se pesaron y midieron el 300 peces del estanque, obteniendo valores promedio de peso y longitud. Se utilizó una balanza digital de $1 \mathrm{~kg}$ y una ictiometro graduado de $30 \mathrm{~cm}$.

Los datos de peso y longitud fueron tratados con el programa spss 17

La cosecha fue por drenado del estanque combinado con captura con redes dentro del estanque. Los parámetros de crecimiento se determinaron mediante la ecuación de crecimiento de Von Bertalanffy, también se determino la relación Talla- Peso.

Análisis estadístico. El procesamiento de datos se realizó bajo las siguiente formula

\section{- Biomasa.}

$B=$ Población $\times$ Peso Promedio del Individuo.

Fuente: Ricker, 1975

- Estimación de los valores de relación peso - talla.

$$
\begin{aligned}
& W_{\alpha}=q * L^{b} \\
& \ln W(i)=\ln (q)+b * \ln (i)
\end{aligned}
$$

- Curva de crecimiento de Von Berttalanffy en peso.

$$
W(t)=q * L^{3}\left(1-\mathcal{E}^{-k\left(t-t_{0}\right)}\right)^{3}
$$

$$
L_{(t)}=L_{\alpha}\left(1-\mathcal{E}^{-k\left(t-t_{0}\right)}\right)
$$

Donde las variables son:

$$
\begin{aligned}
& L_{(t)}-L_{\alpha}\left(1-\mathcal{E}^{-k\left(t-t_{0}\right)}\right) \\
& t_{(l)}=t_{0}-\frac{1}{k} \ln \left(1-\frac{l_{(t)}}{l_{(\alpha)}}\right) \\
& t_{0}=\frac{-a}{b}
\end{aligned}
$$

\section{RESULTADOS Y DISCUSIÓN}

El cuadro $\mathrm{N}^{\circ} 1$ muestra los datos obtenidos durante las 22 semanas de muestreos de 300 ejemplares. cuyos datos han sido procesados según la fórmula 3 ,

La velocidad de crecimiento semanal total durante la fase de cultivo de 22 semanas fue de $17.29 \%$ o de 7.66 gr por semana llevados a crecimiento diario $1.09 \mathrm{gr}$. A una densidad de 5 peces $/ \mathrm{m}^{2}$. Valores muy superiores a los obtenidos por Gómez et al 2010, de crecimientos diarios de 0,47 gr /día con ejemplares iniciales de 1 gr de peso y finales de $71 \mathrm{gr}$ después de 6 meses.

\section{Relación Talla - Peso}

Se hizo el análisis estadístico de la talla y el peso en el cuadro $\mathrm{N}^{\circ} 02$ y se encontró que el modelo que se ajustó mejor a los datos fue de tipo potencial, el cual se expresó como $\mathrm{W} \alpha=\mathbf{0 . 0 4 3}{ }^{*} \mathrm{~L}^{2.681}$ con una correlación entre $\mathrm{L}(\mathrm{i})$ y W(i) de $97 \%$ $\mathrm{p}<0.001$ (Fig.1). La prueba de " $t$ " de student indicó que el valor del exponente $b=2.681$ no fue significativamente diferente al valor de 3 $(t=6.314, p>0.05)$, por lo que el crecimiento de la especie de este estanque se consideró como de tipo isométrico. 
Tabla 1: Controles biometricos y parametros de producción de Oreochromis niloticus TILAPIA DEL NILO (GRIS).

\begin{tabular}{ccccc}
\hline Fecha & $\begin{array}{c}\text { Peso promedio } \\
(\mathrm{gr})\end{array}$ & $\begin{array}{c}\text { Long. Promedio } \\
(\mathrm{cm})\end{array}$ & $\begin{array}{c}\text { Tamaño de muestra } \\
\left(\mathrm{N}^{\circ} \text { peces }\right)\end{array}$ & $\begin{array}{c}\text { Biomasa } \\
\text { KG }\end{array}$ \\
\hline 01 -may-11 & 3,8380 & 6 & 300 & 1,1514 \\
\hline 08 -may-11 & 9,2350 & 7,5 & 300 & 2,7705 \\
\hline 15 -may-11 & 16,4613 & 9 & 300 & 4,9384 \\
\hline 22 -may-11 & 22,5657 & 10 & 300 & 6,7697 \\
\hline 29 may-11 & 29,0560 & 11 & 300 & 8,7168 \\
\hline 05 -jun-11 & 38,6327 & 12 & 300 & 11,5898 \\
\hline 12 -jun-11 & 52,6893 & 13,5 & 300 & 15,8068 \\
\hline 19 -jun-11 & 64,1557 & 14 & 300 & 19,2467 \\
\hline 26 -jun-11 & 79,4193 & 16 & 300 & 23,8258 \\
\hline 03 -jul-11 & 85,1807 & 17 & 300 & 25,5542 \\
\hline 10 -jul-11 & 101,45 & 18 & 300 & 30,435 \\
\hline 17 -jul-11 & 112,76 & 19 & 300 & 33,828 \\
\hline 24 -jul-11 & 125,34 & 19,5 & 300 & 37,602 \\
\hline 31-jul-11 & 129,54 & 20 & 300 & 38,862 \\
\hline 07 -ago-11 & 138,7 & 20 & 300 & 41,61 \\
\hline 14 -ago-11 & 142,28 & 21 & 300 & 42,684 \\
\hline 21 -ago-11 & 147,18 & 21 & 300 & 44,154 \\
\hline 28 -ago-11 & 149,69 & 21 & 300 & 44,907 \\
\hline 04 -set-11 & 157,2 & 21 & 300 & 47,16 \\
\hline 11 -set-11 & 159,3 & 22 & 300 & 4,79 \\
\hline 18 -set-11 & 164,23 & 22 & 300 & \\
\hline 25 -set-11 & 172,4 & 24 & 300 & \\
\hline
\end{tabular}

\section{Relación Talla- Peso}

$$
W_{\alpha}=q * L^{b}
$$

$$
\mathrm{W} \alpha=0.043^{*} \mathrm{~L}^{2.681}
$$

Lo que indica que el crecimiento obtenido es alométrico negativo con 2.86, mientras que Gómez et al 2010, determino un crecimiento alometrico positivo de 3.099. El valor del exponente $b=2.681$ no fue significativamente diferente al valor de 3 , por lo que el crecimiento de la especie de este embalse se consideró como de tipo isométrico. Esto se denota en el grafico de Longitud - peso calculado en el cual la curva sigue un modelo exponencial de crecimiento paralelo entre peso y longitud. 
Tabla 2: Relación Talla Peso de la Tilapia nilotica

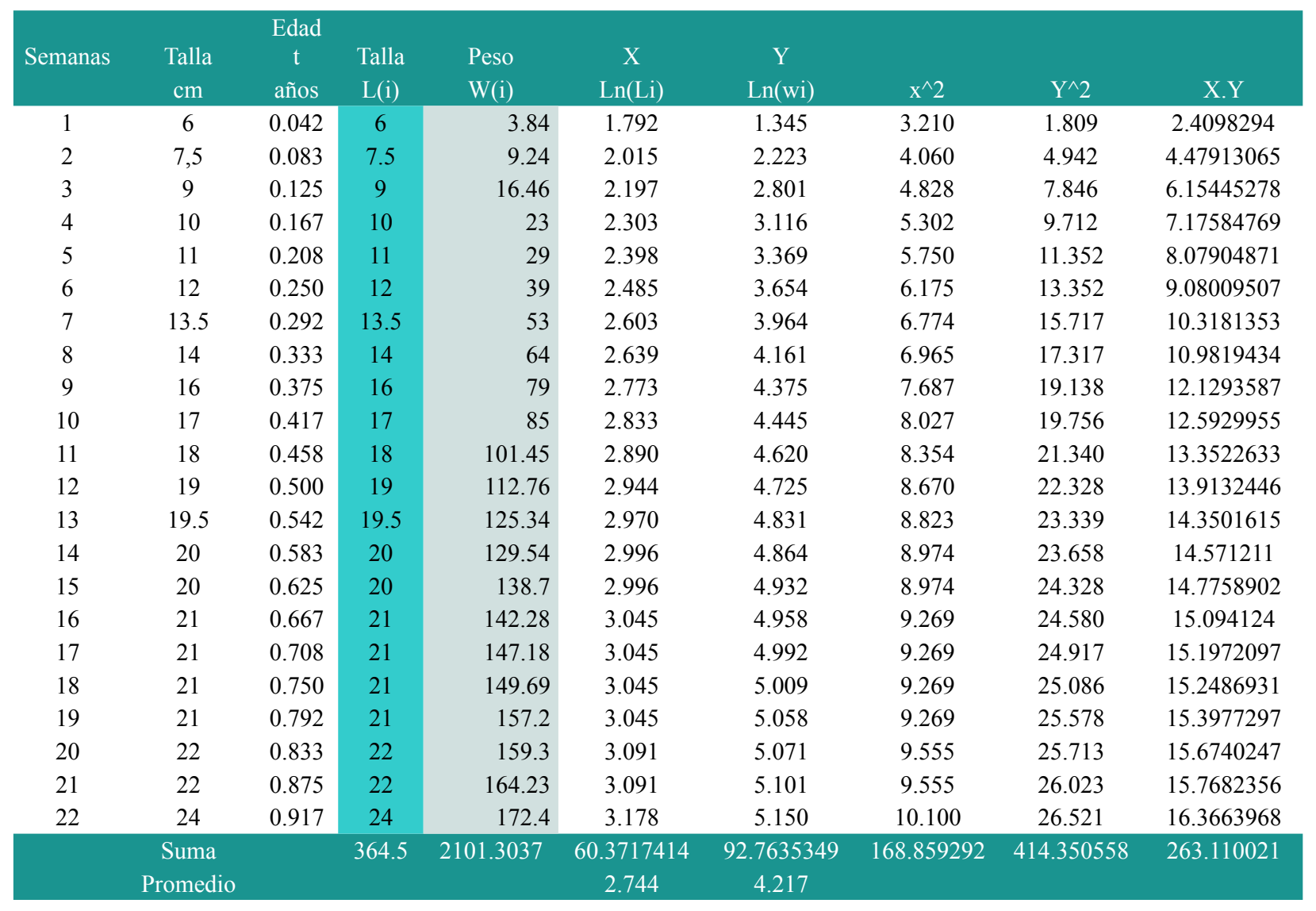

$\begin{array}{cccc} & \mathrm{a} & \mathrm{b} & \mathrm{q} \\ \text { Estanque 1 } & -3.142 & 2.681 & 0.043\end{array}$

Linealizando la relación talla-peso por medio de logaritmos obtenemos los parámetro de condición determinando que la relación Talla Peso esta dada por la siguiente fórmula

$$
\text { Gráfico 1: Relación longitud - peso calculado }
$$

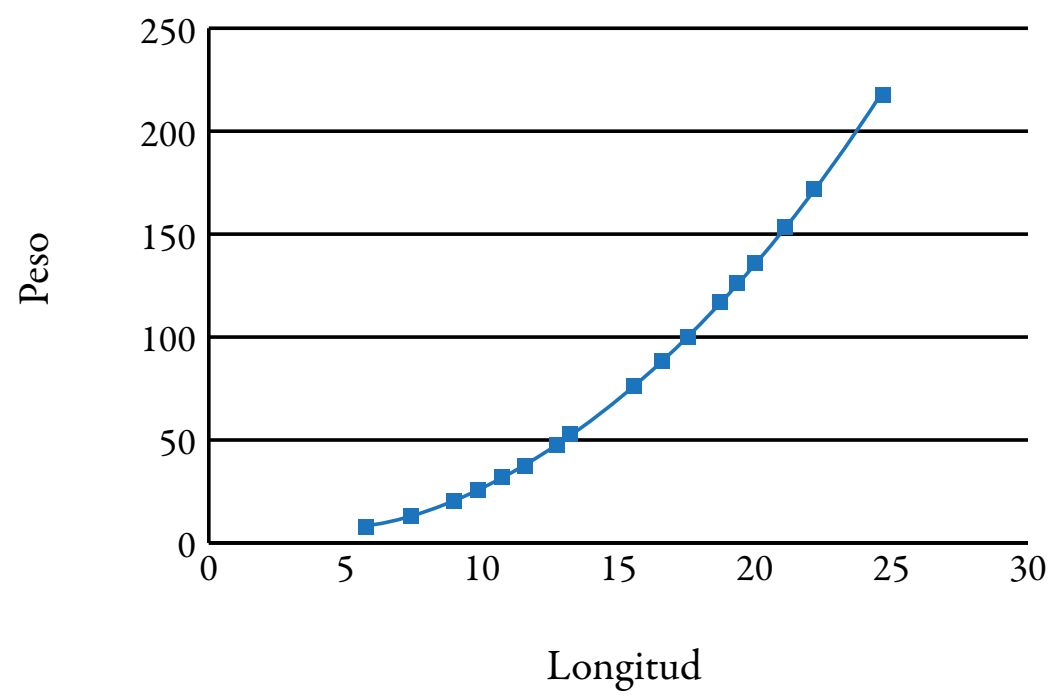


Tabla 3 : Longitud - peso calculado con los parámetros del análisis relación peso - longitud real.

\begin{tabular}{|cc|}
\hline Talla & Peso calculado \\
\hline L(i) & W(i) \\
\hline 6 & 5.2443535 \\
\hline 7.5 & 9.53910661 \\
\hline 9 & 15.5522283 \\
\hline 10 & 20.6285383 \\
\hline 11 & 26.6343587 \\
\hline 12 & 33.6320528 \\
\hline 13.5 & 46.1204238 \\
\hline 14 & 50.8437669 \\
\hline 16 & 72.7300907 \\
\hline 17 & 85.5661544 \\
\hline 18 & 99.7364812 \\
\hline 19 & 115.294041 \\
\hline 19.5 & 123.609329 \\
\hline 20 & 132.29087 \\
\hline 20 & 132.29087 \\
\hline 21 & 150.778141 \\
\hline 21 & 150.778141 \\
\hline 21 & 150.778141 \\
\hline 21 & 150.778141 \\
\hline 22 & 170.806213 \\
\hline 22 & 170.806213 \\
\hline 24 & 215.682443 \\
\hline
\end{tabular}

\section{Parámetros de crecimiento}

Utilizando el análisis estadístico del cuadro No 04 y la ecuación inversa de Von Berttalanffy, se calcularon los valores de $\mathrm{K}, \mathrm{L} \infty$, To, los cuales nos llevaron a obtener la siguiente ecuación Ecuación de crecimiento de Von Berttalanffy en talla

$$
L_{(t)}=29.930\left(1-\mathcal{E}^{-1.536(t+0.132)}\right)
$$

$\begin{array}{ccccccc} & \mathrm{L} & \mathrm{K} & \mathrm{To} & \mathrm{q} & \mathrm{b} & \mathrm{W} \\ \begin{array}{c}\text { Estanque } \\ 1\end{array} & 29.930 & 1.536 & -0.132 & 0.043 & 2.681 & 392.32\end{array}$


Tabla 4: Determinación del parámetro de crecimiento To, con la ecuación inversa de Von Berttalanffy, $\mathrm{L} \alpha=29.930 \mathrm{~cm}$

\begin{tabular}{|c|c|c|c|c|c|c|c|}
\hline \multirow[t]{2}{*}{ Semanas } & \multicolumn{7}{|c|}{$-\ln \left(1-\frac{l(t)}{l(\alpha)}\right)$} \\
\hline & $\mathrm{cm}$ & $\operatorname{edad}(t)$ & $\mathrm{L}(\mathrm{t})$ & $\mathrm{Y}$ & $x^{\wedge} 2$ & $\mathrm{Y}^{\wedge} 2$ & X.Y \\
\hline 1 & 6 & 0.042 & 6 & 0.224 & 0.002 & 0.050 & 0.009 \\
\hline 2 & 7,5 & 0.083 & 7.5 & 0.288 & 0.007 & 0.083 & 0.024 \\
\hline 3 & 9 & 0.125 & 9 & 0.358 & 0.016 & 0.128 & 0.045 \\
\hline 4 & 10 & 0.167 & 10 & 0.407 & 0.028 & 0.165 & 0.068 \\
\hline 5 & 11 & 0.208 & 11 & 0.458 & 0.043 & 0.210 & 0.095 \\
\hline 6 & 12 & 0.250 & 12 & 0.512 & 0.063 & 0.263 & 0.128 \\
\hline 7 & 13.5 & 0.292 & 13.5 & 0.600 & 0.085 & 0.360 & 0.175 \\
\hline 8 & 14 & 0.333 & 14 & 0.631 & 0.111 & 0.398 & 0.210 \\
\hline 9 & 16 & 0.375 & 16 & 0.765 & 0.141 & 0.585 & 0.287 \\
\hline 10 & 17 & 0.417 & 17 & 0.839 & 0.174 & 0.704 & 0.350 \\
\hline 11 & 18 & 0.458 & 18 & 0.920 & 0.210 & 0.846 & 0.422 \\
\hline 12 & 19 & 0.500 & 19 & 1.007 & 0.250 & 1.015 & 0.504 \\
\hline 13 & 19.5 & 0.542 & 19.5 & 1.054 & 0.293 & 1.111 & 0.571 \\
\hline 14 & 20 & 0.583 & 20 & 1.103 & 0.340 & 1.217 & 0.644 \\
\hline 15 & 20 & 0.625 & 20 & 1.103 & 0.391 & 1.217 & 0.690 \\
\hline 16 & 21 & 0.667 & 21 & 1.209 & 0.444 & 1.463 & 0.806 \\
\hline 17 & 21 & 0.708 & 21 & 1.209 & 0.502 & 1.463 & 0.857 \\
\hline 18 & 21 & 0.750 & 21 & 1.209 & 0.563 & 1.463 & 0.907 \\
\hline 19 & 21 & 0.792 & 21 & 1.209 & 0.627 & 1.463 & 0.957 \\
\hline 20 & 22 & 0.833 & 22 & 1.328 & 0.694 & 1.764 & 1.107 \\
\hline 21 & 22 & 0.875 & 22 & 1.328 & 0.766 & 1.764 & 1.162 \\
\hline 22 & 24 & 0.917 & 24 & 1.619 & 0.840 & 2.620 & 1.484 \\
\hline & suma & 10.5416667 & 364.5 & 19.3820444 & 6.58854167 & 20.3513141 & 11.500676 \\
\hline & promedio & 0.479 & & 0.881 & & & \\
\hline
\end{tabular}

Aplicando la formula de la Ecuación de crecimiento de Von Berttalanffy transformada en peso, se puede asumir que el peso asintótico ha alcanzar a las condiciones de cultivo evaluadas es de $392.32 \mathrm{gr}$, con un talla infinita de $29.930 \mathrm{~cm}$ (ver cuadro 5)

\section{DISCUSIÓN}

El análisis de la relación talla $\mathrm{L}(\mathrm{i})$ Peso W(i), mostró un crecimiento de tipo isométrico en el cual los organismos presentan un crecimiento proporcional entre la talla y peso. Este mismo tipo de crecimiento ha sido reportado por Flores
(2006) y Beltrán-Álvarez et al.(1997). Estudios realizados en distintas especies de tilapias muestran que los valores de la pendiente de esta relación oscila entre 2.5 y 3.5 (Granado 1996). Esto indica que los valores obtenidos para las tilapias del estanque se encuentra dentro de este intervalo, y da confiabilidad a los resultados obtenidos en el presente estudio

Los parámetros de crecimiento de Von Berttalanffy, corresponden para un $\mathrm{L} \alpha$ $29.930 \mathrm{~cm}$, un peso asintótico de $\mathrm{W} \alpha$ de 392.32 gr, con un velocidad de crecimiento K de 1.536 ,una edad To de -0.136 año, los que se asemejan 
Tabla 5:

\begin{tabular}{|rr|}
\hline $\mathrm{T}$ & \multicolumn{1}{c|}{$\mathrm{L}_{\text {calculado }}$} \\
\hline 0.25 & 4.96154266 \\
\hline 0.50 & 12.923199 \\
\hline 0.75 & 18.3461334 \\
\hline 1.00 & 22.0398644 \\
\hline 1.25 & 24.5557807 \\
\hline 1.50 & 26.2694503 \\
\hline 1.75 & 27.4366846 \\
\hline 2.00 & 28.2317245 \\
\hline 2.25 & 28.7732512 \\
\hline 2.50 & 29.142102 \\
\hline 2.75 & 29.3933379 \\
\hline 3.00 & 29.5644626 \\
\hline 3.25 & 29.681021 \\
\hline 3.50 & 29.7604126 \\
\hline
\end{tabular}

Gráfico 2:

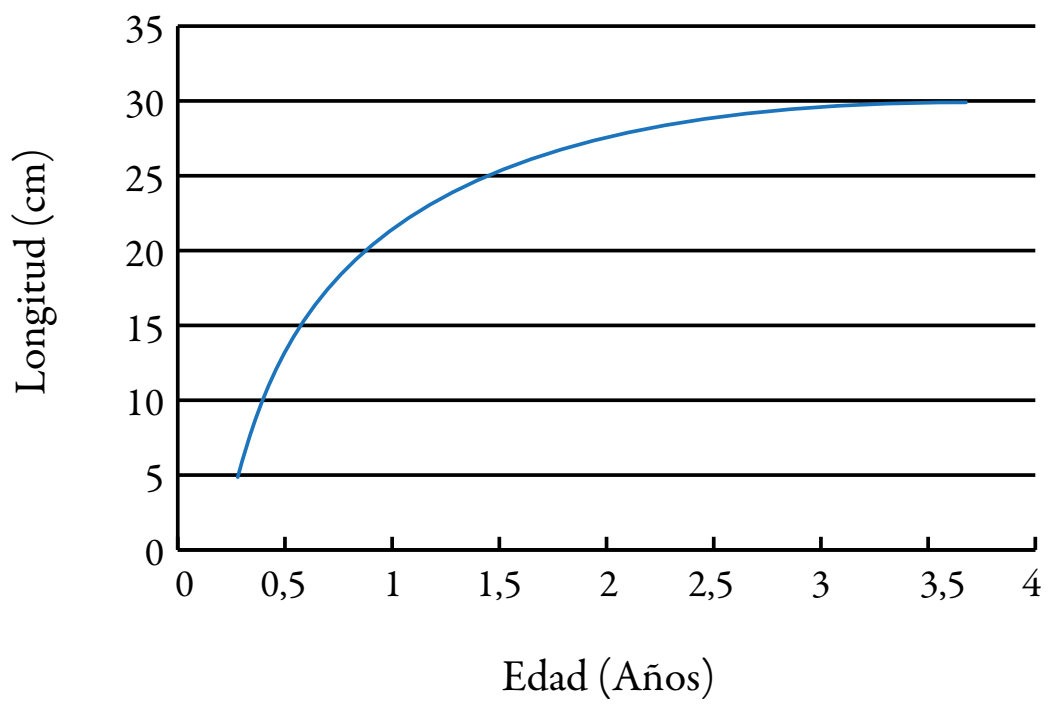

con lo reportado por Pérez \& Patlani (2002) registraron para hembras una $\mathrm{L} \infty=21.15 \mathrm{~cm}$, $\mathrm{W} \infty=156.89 \mathrm{~g}$ y para machos una $\mathrm{L} \infty=56.56 \mathrm{~cm}$, $\mathrm{W} \infty=2972.54 \mathrm{~g}$ y una $\mathrm{k}=0.13$, en la variación de estos valores en comparación con los del presente estudio, también influyen las longitudes máximas registradas por dichos autores

\section{REFERENCIAS BIBLIOGRÁFICAS.}

1. Beltrán-Álvarez, R., P.J. Sánchez, G.L. Valdez \& A.O. Salas. 2009. Edad y crecimiento de la mojarra Oreochromis aureus (Pisces: Cichlidae) en la Presa Sanalona, Sinaloa, México. Rev. Biol. Trop. 58: 325-338. 
2. Beverton, R.J.H. \& S.J. Holt. 1957. On the dynamics of exploited fish populations. In Erzini et al. 1989. Microcomputer Application in Fisheries. Instituto Técnologico y de Estudios Superiores de Monterrey Campus Guaymas Sonora, México.

3. Campana, S.E. \& J.D. Neilson. 1985. Microstructure of fish otoliths. Can. J. Fish. Aquat. Sci. 42: 1014-1032. Carlander, K.D. 1982. Standard intercepts for calculating lengths from scale measurements for some centrarchid and percid fishes. Trans. Amer. Fish. Soc. 111: 332-336.
4. Jiménez-Badillo, L. 2006. Age-growth models for tilapia Oreochromis aureus (Perciformes, Cichlidae) of the Infiernillo reservoir, Mexico and reproductive behaviour. Rev. Biol. Trop. 54: 277-588.

5. Von Bertalanffy, L. 1938. A quantitative theory of organic growth (Inquires on growth Iasw II) Hum. Biol. 10:181-213. 